\title{
A LATTICE-POINT PROBLEM. II
}

\author{
BY \\ BURTON RANDOL( $\left.{ }^{(}\right)$
}

In this paper, which depends heavily on [3], we shall discuss the $n$-dimensional analogue of the example considered in [4], and in $\$ 2$ of [3]. Our main result is as follows:

THEOREM 1. Suppose $k$ is an integer $\geqq 1$, and denote by $V$ the volume of the region in $n$-space defined by $y_{1}^{2 k}+\cdots+y_{n}^{2 k} \leqq 1$. Define numbers $A, B$, and $R$ by setting $A=(2 k-1)(n-1) / 4 k^{2}, B=n(n-1) /(2 k n+2 k), R=\max (A, B)$. Let $N(x)$ be the number of integral lattice-points $\left(m_{1}, \ldots, m_{n}\right)$, satisfying $m_{1}^{2 k}+\cdots+m_{n}^{2 k} \leqq x$. Then $N(x)=V x^{n / 2 k}+O\left(x^{R}\right)$. Moreover, if $A>B$, then this estimate is the best possible. I.e., in this case, $N(x)=V x^{n / 2 k}+\Omega\left(x^{R}\right)$.

The proof of Theorem 1 depends on the following theorem, which may be of independent interest:

TheOREM 2. For any nonzero vector $X=|X|\left(x_{1}^{*}, \ldots, x_{n}^{*}\right)$, define $A(X)$ to be the product of the nonzero $x_{i}^{*}$ 's (here " $|X|$ " denotes the length of $X$ ), and suppose $\partial C$ is defined as in [3], i.e., $\partial C=$ the graph of $y_{1}^{2 k}+\cdots+y_{n}^{2 k}=1$. Then there exists a positive integer $h$, depending only on $k$ and $n$, and having the following property: for any function $f(Y)\left(Y=\left(y_{1}, \ldots, y_{n}\right)\right)$ which is $C^{h}$ in a neighborhood of $\partial C$, there is a constant $M(f)>0$, depending only on bounds for $f(Y)$ and its derivatives of order $\leqq h$ in a neighborhood of $\partial C$, such that if $X=|X|\left(x_{1}^{*}, \ldots, x_{n}^{*}\right)$ is a nonzero vector, for which exactly $j$ of the $x_{i}^{*}$ 's vanish, then

$$
\left|\int_{\partial C} f(Y) e^{2 \pi i(X, Y)} d S_{Y}\right| \leqq M(f)(A(X))^{-(k-1) /(2 k-1)}|X|^{-\alpha_{j}},
$$

where $\alpha_{j}=j / 2 k+(n-j-1) / 2$, and $d S_{Y}=$ the area element on $\partial C$.

Since the proof of Theorem 2 is somewhat tedious, we shall assume the correctness of this theorem for the time being, and proceed with the proof of Theorem 1. Throughout the following the terminology is, for the most part, that of [3], with which we assume some familiarity. For convenience, however, we recall briefly a few of the definitions in [3].

Received by the editors March 9, 1966.

( ${ }^{1}$ This research was partially supported by the National Science Foundation under Grant No. NSF-GP-1814. 
1. $C=\left\{\left(y_{1}, \ldots, y_{n}\right) \mid y_{1}^{2 k}+\cdots+y_{n}^{2 k} \leqq 1\right\}$. (Recall that we have already defined $\partial C$ to be the set $\left\{\left(y_{1}, \ldots, y_{n}\right) \mid y_{1}^{2 k}+\cdots+y_{n}^{2 k}=1\right\}$.)

2. $n(Y)=$ the outward normal to $\partial C$, at the point $Y$ on $\partial C$.

3. $J(x, Y)=$ the characteristic function of the set

$$
\left\{\left(y_{1}, \ldots, y_{n}\right) \mid y_{1}^{2 k}+\cdots+y_{n}^{2 k} \leqq x\right\} .
$$

4. $\hat{J}(x, Y)=$ the Fourier transform of $J(x, Y)$ with respect to the second variable.

5. $\left\{\delta_{\varepsilon}(Y)\right\}=$ a family of $C^{\infty}$ approximate identities with respect to convolution.

6. $J_{\varepsilon}(x, Y)=$ the convolution of $J(x, Y)$ and $\delta_{\varepsilon}(Y)$.

7. $N_{\varepsilon}(x)=\sum J_{\varepsilon}(x, N)=\sum \delta_{\varepsilon}(N) \hat{J}(x, N)$, where the caret denotes the Fourier transform, and the summation is over all integral lattice-points in $E^{n}$. (The second equality is a consequence of the Poisson summation formula.)

8. For any nonzero vector $Y, \beta(Y)=Y /|Y|$.

Finally, we recall the explicit formula for $\hat{J}(x, Y)$ given in [3]:

$$
\hat{J}(x, Y)=\frac{x^{(n-1) / 2 k}}{2 \pi i|Y|} \int_{\partial C} e^{2 \pi i x^{1 / 2 k(Y, Z)}}(\beta(Y), n(Z)) d S_{Z} .
$$

Proof of Theorem 1. In order to apply a result from [3] which we will require, it is desirable that the form $y_{1}^{2 k}+\cdots+y_{n}^{2 k}$ have a high degree, depending on $\partial C$, of homogeneity. If this is not initially the case, we can, as in [3], bring about the desired effect by treating the corresponding lattice-point problem for

$$
\left(y_{1}^{2 k}+\cdots+y_{n}^{2 k}\right)^{w}
$$

where $w$ is large, and then deducing the desired error term from the one we obtain for this case, by the substitution of $x^{w / 2 k}$ for $x$. To avoid trivial complications, we shall assume from the outset that the result from [3] applies directly.

Define

9. $z=x^{(k n-n+k) /(k n+k)}$.

10. $p=[(n+1) / 2]$.

11. For each function $f(x)$, integrable over finite subintervals of $[0, \infty)$, set

$$
\mathrm{I} f(x)=\int_{x}^{x+z} d x_{1} \cdots \int_{x_{p-1}}^{x_{p-1}+z} f\left(x_{p}\right) d x_{p} \quad\left(x_{0}=x\right) .
$$

Now it follows immediately from [3], that

$$
\mathrm{I} N_{\varepsilon}(x)=V \mathrm{I} x^{n / 2 k}+\Sigma^{\prime} \delta_{\varepsilon}(N) \mathrm{I} \hat{J}(x, N),
$$

with the series on the right absolutely convergent. (Here the operator I on the right-hand side is, of course, applied in the first variable, and the prime on the summation sign means that the sum is carried out over all nonzero integral lattice-points.) 
Now $V \mathrm{I} x^{n / 2 k}=V z^{p} \xi^{n / 2 k}$, for some $\xi$ between $x$ and $x+p z$, and by the mean value theorem, $\xi^{n / 2 k}-x^{n / 2 k}=O\left(z x^{(n / 2 k)-1}\right)$. Thus,

$$
V \mathrm{I} x^{n / 2 k}=V z^{p} x^{n / 2 k}+O\left(z^{p+1} x^{(n / 2 k)-1}\right)=V z^{p} x^{n / 2 k}+O\left(z^{p} x^{B}\right),
$$

so (2) can be written

$$
\text { I } N_{\varepsilon}(x)=V z^{p} x^{n / 2 k}+O\left(z^{p} x^{B}\right)+\sum^{\prime} \delta_{\varepsilon}(N) \operatorname{I} \hat{J}(x, N) .
$$

Again by [3], we note that $\lim _{\varepsilon \rightarrow 0}$ I $N_{\varepsilon}(x)$ exists, and equals $\mathrm{I} N(x)$.

Imitating now the treatment in $\$ 2$ of [3], we split the sum on the right-hand side of (3) into $n$ parts

$$
\sum_{(0)} \hat{\delta}_{\varepsilon}(N) \mathrm{I} \hat{J}(x, N)+\cdots+\sum_{(n-1)} \hat{\delta}_{\varepsilon}(N) \mathrm{I} \hat{J}(x, N),
$$

where the subscript $(j)$ on a summation sign means that the sum is taken over precisely those integral lattice-points for which exactly $j$ of the coordinates are zero.

We now require an estimate for $\sum_{(j)} \delta_{\varepsilon}(N)$ I $\hat{J}(x, N)(j=0, \ldots, n-1)$, and to simplify matters, we can clearly suppose, in estimating $\sum_{(j)} \delta_{\varepsilon}(N) \mathrm{I} \hat{J}(x, N)$, that $N$ ranges over the integral lattice-points in the subset $E_{*}^{n-j}$ of $E^{n}$, consisting of exactly those points for which none of the first $n-j$ coordinates, and all of the last $j$ coordinates, vanish. In order to get a good estimate, we need two estimates for I $\hat{J}(x, N)$. We obtain the first by applying the version of Lemma 1 of [3], which corresponds to the constants provided by Theorem 2 of this paper (see [3] for details). The result is: $\mathrm{I} \hat{J}(x, N)=O\left(F_{1 j}(x, N)\right)$, where

$$
F_{1 j}(x, N)=(A(N))^{-(k-1) /(2 k-1)} z^{p} x^{\left(n-1-\alpha_{j}\right) / 2 k}|N|^{-\left(1+\alpha_{j}\right)}
$$

and the estimate is uniform in $x$ and $N$. To obtain a second estimate, we apply the corresponding analogue of Lemma 2 of [3]. The result is: I $\hat{J}(x, N)=O\left(F_{2 j}(x, N)\right)$, uniformly in $x$ and $N$, where

$$
F_{2 j}(x, N)=(A(N))^{-(k-1) /(2 k-1)} x^{(n+2 k p-p-1-\alpha j) / 2 k}|N|^{-\left(1+p+\alpha_{j}\right)}
$$

We return now to the question of estimating $\sum_{(j)} \delta_{\varepsilon}(N) \mathrm{I} \hat{J}(x, N)$. Define $r=(n-1) /(2 k n+2 k)$. Now since $\left|\hat{\delta}_{\varepsilon}(N)\right| \leqq 1$ for all $N$, we have

$$
\left|\sum_{(j)} \delta_{\varepsilon}(N) \operatorname{I} \hat{J}(x, N)\right| \leqq M\left[\sum_{|N| \leqq x^{r}} F_{1 j}(x, N)+\sum_{|N|>x^{r}} F_{2 j}(x, N)\right],
$$

for some $M>0$, with the sums taken over the indicated lattice-points in $E_{*}^{n-j}$. Consider now the first sum on the right-hand side. A little reflection shows that it can be dominated, for large $x$, by a fixed multiple of

$$
z^{p} x^{\left(n-1-\alpha_{j}\right) / 2 k} \int(A(Y))^{-(k-1) /(2 k-1)}|Y|^{-\left(1+\alpha_{j}\right)} d V_{Y}
$$


where $d V_{Y}$ is the volume element in $E_{*}^{n-j}$, and the integration is taken over the subset of $E_{*}^{n-j}$ defined by $1 \leqq|Y| \leqq x^{r}$. Now $(A(Y))^{-(k-1) /(2 k-1)}$ is homogeneous of weight zero, so we can write the last integral in polar coordinates as

$$
z^{p} x^{\left(n-1-\alpha_{j}\right) / 2 k} \int_{S_{\bullet}^{n-1-1}}(A(\varphi))^{-(k-1) /(2 k-1)} d S_{\varphi} \int_{\rho=1}^{x^{r}} \rho^{n-j-1-\left(1+\alpha_{j}\right)} d \rho
$$

where $S_{*}^{n-j-1}$ denotes $S^{n-j-1}$, minus the points for which a rectangular coordinate vanishes, and $\rho=|Y|, \varphi=\left(y_{1} / \rho, \ldots, y_{n-j} / \rho\right)$. But the integral of $(A(\varphi))^{-(k-1) /(2 k-1)}$ over $S_{*}^{n-j-1}$ is finite, so for large $x$, the first sum can be dominated by some fixed multiple of

$$
z^{p} x^{\left(n-1-\alpha_{j}\right) / 2 k} \int_{\rho=1}^{x^{r}} \rho^{n-j-1-\left(1+\alpha_{j}\right)} d \rho .
$$

Now if it should be the case that $n-j<1+\alpha_{j}$ (this will certainly be true for some $j$ 's, beginning with $j=n-1)$, then it follows from (4) that $\sum F_{1 j}(x, N)$ is absolutely convergent, with the sum taken over all lattice-points in $E_{*}^{n-j}$, and

$$
\sum F_{1 j}(x, N)=O\left(z^{p} x^{b(j)}\right)
$$

where $b(j)=\left(n-1-\alpha_{j}\right) / 2 k$. If $n-j<1+\alpha_{j}$, we shall take (5) as our estimate for $\sum_{(j)} \delta_{\varepsilon}(N) I \hat{J}(x, N)$. If, on the other hand, $n-j \geqq 1+\alpha_{j}$, we see from (4) that if $n-j=1+\alpha_{j}$,

$$
\sum_{|N| \leqq x^{r}} F_{1 j}(x, N)=O\left(z^{p} x^{b(j)} \log x\right)
$$

while for $n-j>1+\alpha_{j}$,

$$
\sum_{|N| \leqq x^{r}} F_{1 j}(x, N)=O\left(z^{p} x^{c(j)}\right)
$$

where $c(j)=\left(2 n^{2}-2 n-2 n \alpha_{j}-n j+j\right) /(2 k n+2 k)$. (Note that the case $n-j=1+\alpha_{j}$ only occurs if $(k n-k) /(k+1)$ is an integer.)

By going through a similar process for the sum

$$
\sum_{|N|>x^{r}} F_{2 j}(x, N)
$$

we obtain the estimate

$$
\sum_{|N|>x^{r}} F_{2 j}(x, N)=O\left(z^{p} x^{c(j)}\right)
$$

for all $j$ from 0 to $n-1$. (In this case, the values of $j$ for which $n-j \leqq 1+\alpha_{j}$ create no special problem.) 
Before summarizing, we note that if $j_{0}=(k n-k) /(k+1)$ is an integer, then $j_{0}<n-1$. Moreover, $b\left(j_{0}\right)=c\left(j_{0}\right)$, and since $b(j)$ is an increasing function, $x^{b\left(j_{0}\right)} \log x$ is of a lower order of magnitude than $x^{b\left(j_{0}+1\right)}$.

Now combining results, we see that

$$
\begin{aligned}
\sum_{(j)} \delta_{\varepsilon}(N) \mathrm{I} \hat{J}(x, N) & =O\left(z^{p} x^{b(j)}\right) & & \text { if } n-j<1+\alpha_{j} \\
& =o\left(z^{p} x^{b(j+1)}\right) & & \text { if } n-j=1+\alpha_{j} \\
& =O\left(z^{p} x^{c(j)}\right) & & \text { if } n-j>1+\alpha_{j}
\end{aligned}
$$

Thus, if we note that the functions $b(j)$ and $c(j)$ are linear, and satisfy $b(O)<$ $c(O)=B, c(n-1)<b(n-1)=A$, we immediately obtain that

$$
\sum^{\prime} \delta_{\varepsilon}(N) \mathrm{I} \hat{J}(x, N)=O\left(z^{p} x^{R}\right)
$$

where the summation is over all nonzero lattice-points in $E^{n}$. By (3), this implies that

$$
\text { I } N_{\varepsilon}(x)=V z^{p} x^{n / 2 k}+O\left(z^{p} x^{R}\right)
$$

and letting $\varepsilon \rightarrow 0$, we obtain

$$
\mathrm{I} N(x)=V z^{p} x^{n / 2 k}+O\left(z^{p} x^{R}\right)
$$

Now $N(x)$ is nondecreasing, so

$$
z^{p} N(x) \leqq \mathrm{I} N(x) \leqq z^{p} N(x+p z)
$$

By the first half of this inequality, we find that $N(x) \leqq V x^{n / 2 k}+O\left(x^{R}\right)$ and, making the substitution $y=x+p z$, we easily obtain from the second half that $N(y) \geqq$ $V y^{n / 2 k}+O\left(y^{R}\right)$. Taken together, these last two inequalities prove Theorem 1, except for the statement that if $A>B$, then the result is the best possible. We will now briefly sketch a proof of this last statement. For this, we require some information about $\hat{J}(x, N)$.

Suppose the lattice-point $N$ lies on a coordinate axis $(N \neq 0)$. Then the explicit formula (1), and a standard application of the method of stationary phase [1, pp. 51-56], shows that

$$
\hat{J}(x, N)=K x^{A} \psi(x,|N|)+O\left[x^{A-1 / 2 k}|N|^{-(2 k+n) / 2 k}\right]
$$

where $K$ is a constant, and

$$
\psi(x,|N|)=|N|^{-(2 k+n-1) / 2 k} \sin \left(2 \pi|N| x^{1 / 2 k}-(n-1) \pi / 4 k\right) .
$$


Moreover, the error term is uniform in $x$ and $N$. Now define $B(t)$ to be the continuous periodic function

$$
2 n K \sum_{j=1}^{\infty} \psi\left(t^{2 k}, j\right) .
$$

(Note that since the integral of $B(t)$ over a period is zero, there clearly exists some number $t_{0}$, such that $B\left(t_{0}\right)<0$, since $B(t)$ is not identically zero.)

Now by the same reasoning that led to (6), we find that

$$
\text { I } N(x)=V z^{p} x^{n / 2 k}+\mathrm{I} x^{A} B\left(x^{1 / 2 k}\right)+o\left(z^{p} x^{A}\right) .
$$

But by $(7), z^{p} N(x) \leqq \mathrm{I} N(x)$ so

$$
N(x) \leqq V x^{n / 2 k}+z^{-p} \mathrm{I} x^{A} B\left(x^{1 / 2 k}\right)+o\left(x^{A}\right) .
$$

But $B\left(t_{0}\right)<0$, and since the variation of $t^{1 / 2 k}$ over the interval $[x, x+p z]$ tends to zero as $x \rightarrow \infty$, it is clear that if $x$ is large, and $x^{1 / 2 k}$ differs from $t_{0}$ by an integer, then the sum of the last two terms on the right in (9) is actually of the order of $x^{A}$, and this completes the proof of Theorem 1.

Proof of Theorem 2. We may assume without loss of generality that precisely the first $j$ coordinates of $X$ vanish, and that $X$ lies within the cone $W$, consisting of all vectors within $\operatorname{arc} \cos n^{-1 / 2}$ radians of the positive $y_{n}$-axis.

Now $\partial C$ can be regarded, for purposes of integration, as being composed of two "hemispheres," namely, those points whose $y_{n}$ coordinate is $\geqq 0$, and those points whose $y_{n}$ coordinate is $\leqq 0$. Denoting by $A$ the intersection of the hyperplane $y_{n}=0$ with $C$, we see that the contribution to the integral in Theorem 2 which comes from the upper hemisphere is

$$
\begin{aligned}
& \int_{A} f\left(y_{1}, \ldots, y_{n-1}, D\right) m\left(y_{1}, \ldots, y_{n-1}\right) \\
& \cdot \exp \left[2 \pi i|X|\left(x_{1}^{*} y_{1}+\cdots+x_{n-1}^{*} y_{n-1}+x_{n}^{*} D\right)\right],
\end{aligned}
$$

where $m$ represents the distortion of measure arising from the projection of the upper hemisphere onto $y_{n}=0$, and

$$
D\left(y_{1}, \ldots, y_{n-1}\right)=\left(1-y_{1}^{2 k}-\cdots-y_{n-1}^{2 k}\right)^{1 / 2 k} .
$$

Now there is exactly one point $P=\left(p_{1}, \ldots, p_{n}\right)$ on the upper hemisphere at which the normal to $\partial C$ is parallel to $X$, and by taking the gradient of $y_{1}^{2 k}+\cdots+y_{n}^{2 k}$, we see that there is a positive number $c$, which depends continuously on $X$, such that $p_{i}=c\left(x_{i}^{*}\right)^{1 /(2 k-1)}(i=1, \ldots, n)$. (Note that by our assumption about $X$, precisely the first $j$ of the $p_{i}$ 's are zero. Note also that $P$ and $X$ are determined by each other, so that to the requirement that $X$ be in $W$, there corresponds a requirement 
that $P$ be in a certain cone $W^{\prime}$. In particular, there exists $M>0$, such that $p_{n}, x_{n}^{*}>M$, for all $X$ in $W$ and $P$ in $W^{\prime}$.)

Now introduce coordinates $z_{1}, \ldots, z_{n-1}$, by setting $z_{i}=y_{i}-p_{i}(i=1, \ldots, n-1)$, and define

$$
\begin{aligned}
E\left(z_{1}, \ldots, z_{n-1}\right)= & x_{1}\left(z_{1}+p_{1}\right)+\cdots+x_{n-1}\left(z_{n-1}+p_{n-1}\right) \\
& +x_{n} D\left(z_{1}+p_{1}, \ldots, z_{n-1}+p_{n-1}\right) .
\end{aligned}
$$

Then a routine computation shows that the function

$$
\Phi=E\left(z_{1}, \ldots, z_{n-1}\right)-E\left(0, \ldots, 0, z_{j+1}, \ldots, z_{n-1}\right)
$$

has a zero of order $2 k$ in the variables $z_{1}, \ldots, z_{j}$, where by this we mean that all terms of order less than $2 k$ in the Taylor expansion of $\Phi$ (regarded as a function of $z_{1}, \ldots, z_{j}$ ) are zero, while the sum of the $2 k$ th order terms is a positive form. (Recall that $p_{i}, x_{i}=0$ for $i=1, \ldots, j$.)

Suppose now $\varepsilon>0$ is such that

1. For all $X$ in $W$, the set $S_{\varepsilon}$ stays uniformly away from the boundary of $A$, where $S_{\varepsilon}$ is the product of

$$
\left\{\left(z_{1}, \ldots, z_{j}\right) \mid z_{1}^{2}+\cdots+z_{j}^{2} \leqq \varepsilon\right\}
$$

and

$$
\left\{\left(z_{j+1}, \ldots, z_{n-1}\right) \mid z_{j+1}^{2}+\cdots+z_{n-1}^{2} \leqq \varepsilon\right\} .
$$

2. For all $X$ in $W$ for which $x_{1}^{*}, \ldots, x_{j}^{*}=0$, the function $\Phi$, defined as above, is of the form $\Phi^{*}\left(z_{1}, \ldots, z_{n-1}\right)\left(z_{1}^{2}+\cdots+z_{j}^{2}\right)^{k}$, with $\Phi^{*}$ uniformly bounded away from zero, provided $\left(z_{1}, \ldots, z_{n-1}\right)$ is in $S_{\varepsilon}$.

Now suppose $h(t)$ is a $C^{\infty}$ function of $t(-\infty<t<\infty)$ such that $h(t)=1$ for $|t|<\varepsilon / 2$, and $h(t)=0$ for $|t|>3 \varepsilon / 4$, and set

$$
H\left(z_{1}, \ldots, z_{n-1}\right)=h\left(z_{1}^{2}+\cdots+z_{j}^{2}\right) h\left(z_{j+1}^{2}+\cdots+z_{n-1}^{2}\right) .
$$

Then the integral in (10) can be written in the form

$$
\int_{A} H f m \exp [2 \pi i|X| E]+\int_{A}(1-H) f m \exp [2 \pi i|X| E]
$$

To deal with the second integral, note that if it is combined with the integral which corresponds to it, when the process we have described is duplicated for the lower hemisphere, integration by parts with respect to the vertical variable shows that the result will diminish as rapidly as $|X|^{-h}$, the result being uniform in $W$, and if we take $h \geqq(n-1) / 2$, this term can be ignored (cf. [2, p. 767]). 
It remains to deal with the first integral. The treatment of the corresponding integral for the lower hemisphere is the same, so we shall only consider the case at hand. The first integral in (11) can be written in the form

$$
\begin{array}{r}
\int_{z_{j+1}^{2}+\cdots+z_{n-1}^{2} \leqq \varepsilon} \exp \left[2 \pi i|X| E\left(0, \ldots, 0, z_{j+1}, \ldots, z_{n-1}\right)\right] \\
\times \int_{z_{1}^{2}+\cdots+z_{j}^{2} \leqq \varepsilon} H f m \exp [2 \pi i|X| \Phi] .
\end{array}
$$

By a routine application of the method of stationary phase [1, pp. 51-56], we can expand the inner integral in the last expression into an asymptotic series of descending powers of $|X|$, with $-j / 2 k$ the exponent in the first term, and with the exponent in each term equal to that in its predecessor diminished by $1 / 2 k$. We carry this series to the point where the remainder is $O\left(|X|^{-\alpha} j\right)$. The result will consist of, first of all, the remainder term, which is unimportant, since it already diminishes at the desired rate, and secondly, the product of

$$
\exp \left[2 \pi i E\left(0, \ldots, 0, z_{j+1}, \ldots, z_{n-1}\right)\right]
$$

with a finite series of descending powers of $|X|$ of the type previously described, and having smooth functions of $z_{j+1}, \ldots, z_{n-1}$ for coefficients. (The degree of smoothness depends, of course, on the value of $h$.) If, now, we examine the integral with which we are left, and bear in mind that $p_{i}, x_{i}=0(i=1, \ldots, j)$, it becomes clear that to complete the proof of Theorem 2, it would suffice to prove the theorem for general $n$ in the special case in which none of the $x_{i}^{*}$ 's are zero. Accordingly, we shall again take up the proof from the beginning, but with the assumption now that $x_{1}^{*}, \ldots, x_{n}^{*} \neq 0$. We begin as before, and again reduce the problem to the consideration of an integral of the form

$$
\int_{A} H f m \exp [2 \pi i|X| E]
$$

where $X$ is in $W$, and $H$ is constructed as before, so that its support stays well away from the boundary of $A$.

In order to investigate (12), it is convenient to introduce polar coordinates, by setting $r=\left(z_{1}^{2}+\cdots+z_{n-1}^{2}\right)^{1 / 2}, \varphi=\left(\varphi_{1}, \ldots, \varphi_{n-1}\right)$, where $\varphi_{i}=z_{i} / r$. We may, for fixed $X$, or what is the same thing, for fixed $P$, regard $E$ as a function of $r$ and $\varphi$, which we designate interchangeably by $E$ or $E(r, \varphi)$. To investigate the asymptotic behavior of (12) with respect to $|X|$, we need some information about $E$. In what follows, we shall indicate $j$ th order partial derivatives with respect to $r$ by a parenthetical superscript (e.g., $E^{(j)}, E^{(j)}(r, \varphi)$, etc.). In the case of functions of one variable, we shall use the same notation to designate the appropriate derivative with respect to the argument. 
Note now, that it is clear on geometrical grounds that $E^{(1)}(0, \varphi)=0$ for all $\varphi$ in $S^{n-2}$. Note also that it is sufficient to prove Theorem 2 in the special case in which some of the $\left|p_{i}\right|$ 's are smaller than a number $d>0$, which we may choose at our convenience. This follows from the fact that $\partial C$ has uniformly positive curvature at any fixed distance away from its intersection with the coordinate hyperplanes, and by standard techniques, e.g., stationary phase, (12) is uniformly $O\left(|X|^{-(n-1) / 2}\right)$ in this case. (Cf. also [2, pp. 767-769].)

Definition. For a given $P=\left(p_{1}, \ldots, p_{n}\right)$ and $\varphi$, denote by $a_{n}(P, \varphi)$ the $n$th coefficient in the Taylor expansion of $E^{(1)}(r, \varphi)$ in powers of $r$. I.e., $E^{(1)}(r, \varphi)=$ $\sum a_{n}(P, \varphi) r^{n}$. (Note. It is evident after a moment's reflection, that if $P$ is in $W^{\prime}$, then all the $a_{n}(P, \varphi)$ 's can be majorized by the corresponding coefficients of some fixed power series having a positive radius of convergence.)

Lemma 1. There exist positive numbers $M$ and $d$, such that if $P=\left(p_{1}, \ldots, p_{n}\right)$ satisfies any set of conditions of the form $\left|p_{k_{i}}\right| \leqq d$, where $\left\{k_{i}\right\}$ is a nonempty subsequence of $\{1, \ldots, n-1\}$, then $\left|a_{1}(P, \varphi)\right|+\left|a_{2 k-1}(P, \varphi)\right| \geqq M$, for all $\varphi$ in $S^{n-2}$.

Proof. Define $\left\{k_{i}^{\prime}\right\}$ to be the subsequence of $\{1, \ldots, n-1\}$ complementary to $\left\{k_{i}\right\}$; i.e., $\left\{k_{i}^{\prime}\right\}$ is obtained from $\{1, \ldots, n-1\}$ by striking out the elements of $\left\{k_{i}\right\}$. For the moment, we shall suppose that $\left\{k_{i}^{\prime}\right\}$ is not empty.

Recall now that the function $D$, which we have previously defined, takes the form $\left(1-\left(p_{1}+\varphi_{1} r\right)^{2 k}-\cdots-\left(p_{n-1}+\varphi_{n-1} r\right)^{2 k}\right)^{1 / 2 k}$, when written as a function of $P, r$, and $\varphi$. Since $E$ is the sum of $D$ and a term which is linear in $r$, it is clear that $E^{(2)}=D^{(2)}$. Now define $f(t)=t^{1 / 2 k}(t>0)$, and $g=D^{2 k}$. Then $E^{(2)}=f^{(2)}(g)\left(g^{(1)}\right)^{2}$ $+f^{(1)}(g) g^{(2)}$. Since both terms on the right are negative, and since $f^{(1)}(g)$ is uniformly bounded away from zero, we see that $a_{1}(P, \varphi)=E^{(2)}(0, \varphi)$ is at least of the order of magnitude of

$$
g^{(2)}(0, \varphi)=2 k(2 k-1) \sum_{i=1}^{n-1} \varphi_{i}^{2} p_{i}^{2 k-2}
$$

Now

$$
(2 k-1) ! a_{2 k-1}(P, \varphi)=E^{(2 k)}(0, \varphi)=\left.f^{(1)}(g) g^{(2 k)}\right|_{r=0}+\text { (remainder). }
$$

But $\left.f^{(1)}(g) g^{(2 k)}\right|_{r=0}$ is uniformly bounded away from zero for all $P$ and $\varphi$. Moreover, since the remainder consists of a sum of products of derivatives of $f$ and $g$, with all of the latter of order less than $2 k$, it is clear, from the form of the derivatives of $g$, that if $d$ is sufficiently small, there exist $M^{\prime}, e>0$, independent of $\left\{k_{i}\right\}$, such that for $\sum\left|\varphi_{k_{i}^{\prime}}\right| \leqq e$, we have $\left|a_{2 k-1}(P, \varphi)\right| \geqq M^{\prime}$. On the other hand, if $\sum\left|\varphi_{k_{i}^{\prime}}\right|>e$, it follows from the expression for $g^{(2)}(0, \varphi)$, and from the fact that each $\left|p_{k_{i}^{\prime}}\right|>d$, that $\left|a_{1}(P, \varphi)\right|$ is uniformly bounded away from zero, and this establishes the lemma, if we note that the previous reasoning also applies to the case in which $\left\{k_{i}^{\prime}\right\}$ is empty. 
Throughout the following, we shall assume, as we now may, that in addition to being in $W^{\prime}, P$ is such that the conclusion of Lemma 1 is true, for some fixed value of $M$.

LEMMA 2. There exists $M>0$, such that

$$
\left|E^{(2)}(r, \varphi)\right| \geqq M \sum_{i=1}^{n-1} \varphi_{i}^{2}\left(p_{i}+\varphi_{i} r\right)^{2 k-2} .
$$

Proof. In the proof of Lemma 1, we saw that $E^{(2)}=f^{(2)}(g)\left(g^{(1)}\right)^{2}+f^{(1)}(g) g^{(2)}$, and the last expression is at least of the order of magnitude of

$$
g^{(2)}(r, \varphi)=2 k(2 k-1) \sum_{1}^{n-1} \varphi_{i}^{2}\left(p_{i}+\varphi_{i} r\right)^{2 k-2},
$$

which proves the result.

LEMMA 3. There exists $M>0$ such that $\left|E^{(1)}(r, \varphi)\right| \geqq M r \sum_{i=1}^{n-1} \varphi_{i}^{2} p_{i}^{2 k-2}$.

Proof. This follows immediately from Lemma 2, and the facts that:

1. $E^{(2)}(r, \varphi)$ is of one sign.

2. $E^{(1)}(r, \varphi)=\int_{0}^{r} E^{(2)}(t, \varphi) d t=\int_{0}^{r / 3}+\int_{r / 3}^{2 r / 3}+\int_{2 r / 3}^{r}$.

3. Each of the functions $p_{i}+\varphi_{i} r$ is linear so, for each $i$, there is some third of the interval $[0, r]$ in which $\left(p_{i}+\varphi_{i} r\right)^{2 k-2}$ is greater than a fixed multiple of its value at either endpoint.

Definition. For a polynomial $\pi(x)=a_{m} x^{m}+\cdots+a_{1} x+a_{0}$, with real or complex coefficients, define, for $0<r \leqq 1$,

$$
M_{\pi}(r)=\max _{0 \leqq x \leqq r}|\pi(x)|, \quad M_{\pi}^{*}(r)=\left|a_{m}\right| r^{m}+\cdots+\left|a_{1}\right| r+\left|a_{0}\right| .
$$

LEMMA 4. There exists a constant $M>0$, depending only on $m$, such that if $\pi(x)$ is an mth degree polynomial, then $M_{\pi}(r) \geqq M M_{\pi}^{*}(r)$, for $0<r \leqq 1$.

Proof. By the change of variable $x_{1}=x / r$, we may assume $r=1$. Now $M_{\pi}(1)$ and $M_{\pi}^{*}(1)$ are both norms on $C^{m+1}$, if we identify the coefficients of $\pi$ with coordinates in $C^{m+1}$. The result thus follows from the equivalence of norms on $C^{m+1}$.

Definition. For fixed $P$ and small $r$, define $E_{*}^{(1)}(r, \varphi)=\sum_{n=1}^{\infty}\left|a_{n}(P, \varphi)\right| r^{n}$.

LEMMA 5. There exist $M, e>0$, such that for all $P$ under consideration,

$$
\left|E^{(1)}(r, \varphi)\right| \geqq M E_{*}^{(1)}(r, \varphi),
$$

provided $0 \leqq r \leqq e$. 
Proof. By Lemma 1 , there exist $M^{\prime}>0$ such that $\left|a_{1}(P, \varphi)\right|+\left|a_{2 k-1}(P, \varphi)\right| \geqq M^{\prime}$. Thus, for a given $\varphi$, either $\left|a_{1}(P, \varphi)\right| \geqq M^{\prime} / 2$, or $\left|a_{2 k-1}(P, \varphi)\right| \geqq M^{\prime} / 2$. We shall assume that $\varphi$ is such that the latter condition holds. The proof for the remaining case is essentially the same.

Define $\pi(r, \varphi)=\sum_{n=1}^{2 k-1} a_{n}(P, \varphi) r^{n}, \pi^{*}(r, \varphi)=\sum_{n=1}^{2 k-1}\left|a_{n}(P, \varphi)\right| r^{n}$. Then $E^{(1)}(r, \varphi)=$ $\pi(r, \varphi)+O\left(r^{2 k}\right)$, and $E_{*}^{(1)}(r, \varphi)=\pi^{*}(r, \varphi)+O\left(r^{2 k}\right)$, with the error terms uniform in all variables. Thus, if $e$ is small enough, it is clear, since $\left|a_{2 k-1}(P, \varphi)\right| \geqq M^{\prime} / 2$, that there exists $M^{\prime \prime}>0$, such that for $0 \leqq r \leqq e, \pi^{*}(r, \varphi) \geqq M^{\prime \prime} E_{*}^{(1)}(r, \varphi)$. Now by Lemma 4 , there exists $x \in[0, r]$ and $M^{m}>0$, such that

$$
|\pi(x, \varphi)| \geqq M^{m} \pi^{*}(r, \varphi) \geqq M^{\prime \prime} M^{\prime \prime} E_{*}^{(1)}(r, \varphi) .
$$

But for small $e, E^{(1)}(x, \varphi)-\pi(x, \varphi)$ is negligible in comparison with $E_{*}^{(1)}(r, \varphi)$, so $\mid E^{(1)}(x, \varphi)-o\left(E_{*}^{(1)}(r, \varphi) \mid \geqq M^{\prime \prime} M^{m} E_{*}^{(1)}(r, \varphi)\right.$. This implies the existence of an $M>0$, such that $\left|E^{(1)}(x, \varphi)\right| \geqq M E_{*}^{(1)}(r, \varphi)$, and since $\left|E^{(1)}(t, \varphi)\right|$ is an increasing function of $t$, this implies that $\left|E^{(1)}(r, \varphi)\right| \geqq M E_{*}^{(1)}(r, \varphi)$.

LemMa 6. Suppose $m$ is a fixed positive integer. Then there exist $M, e>0$, such that for $j=1, \ldots, m$, and $0 \leqq r \leqq e, r^{j-1}\left|E^{(j)}(r, \varphi)\right| \leqq M\left|E^{(1)}(r, \varphi)\right|$.

Proof. This is clear by considering Taylor series, if we replace $\left|E^{(1)}(r, \varphi)\right|$ by $E_{*}^{(1)}(r, \varphi)$ and recall Lemma 1 . The result then follows from Lemma 5.

LEMMA 7. Suppose $b_{1}, \ldots, b_{n-1}$ are positive numbers. Then

$$
\int_{S^{n-2}}\left(b_{1} \varphi_{1}^{2}+\cdots+b_{n-1} \varphi_{n-1}^{2}\right)^{-(n-1) / 2} d S_{\varphi}=\frac{2 \pi^{(n-1) / 2}}{\Gamma((n-1) / 2)}\left(b_{1} \cdots b_{n-1}\right)^{-1 / 2} .
$$

Proof. Consider the integral

$$
\int_{-\infty}^{\infty} d y_{n-1} \cdots \int_{-\infty}^{\infty} \exp \left[-\left(b_{1} y_{1}^{2}+\cdots+b_{n-1} y_{n-1}^{2}\right)\right] d y_{1}=\pi^{(n-1) / 2}\left(b_{1} \cdots b_{n-1}\right)^{-1 / 2}
$$

In polar coordinates, the integral becomes

$$
\int_{S^{n-2}} d S_{\varphi} \int_{0}^{\infty} r^{n-2} \exp \left[-\left(b_{1} \varphi_{1}^{2}+\cdots+b_{n-1} \varphi_{n-1}^{2}\right) r^{2}\right] d r
$$

The inner integral is a Mellin transform, whose value is

$$
\frac{1}{2} \Gamma((n-1) / 2)\left(b_{1} \varphi_{1}^{2}+\cdots+b_{n-1} \varphi_{n-1}^{2}\right)^{(n-1) / 2},
$$

and the result follows immediately.

We now estimate (12), and we shall suppose, in what follows, that the support of $H$ is small enough so that the conclusion of Lemma 6 is valid within it. There 
are two cases to distinguish, the case in which $n$ is even, and the case in which $n$ is odd. Since the treatment in both cases is similar, we shall only discuss the case in which $n$ is even.

In polar coordinates, (12) becomes

$$
\int_{S^{n-2}} d S_{\varphi} \int_{0}^{\varepsilon} r^{n-2} H f m e^{i|X| E} d r
$$

where $\varepsilon$ is chosen so that the support of $H$ is contained within the ball of radius $\varepsilon$ about $\left(p_{1}, \ldots, p_{n-1}\right)$. If $n>2$, integration by parts shows that

$$
\begin{aligned}
\int_{0}^{\varepsilon} r^{n-2} H f m e^{i|X| E} d r & =\frac{1}{i|X|} \int_{0}^{\varepsilon} \frac{r^{n-2}}{E^{(1)}} H f m d\left(e^{i|X| E}\right) \\
& =-\frac{1}{i|X|} \int_{0}^{\varepsilon} \frac{\partial}{\partial r}\left(\frac{r^{n-2}}{E^{(1)}} H f m\right) e^{i|X| E} d r .
\end{aligned}
$$

Repeating this $(n-2) / 2$ times, and using the fact that $E^{(1)}$ has a first order zero, we finally end up with an expression of the form

$$
\frac{\text { constant }}{|X|^{(n-2) / 2}} \int_{0}^{\varepsilon} \frac{\partial}{\partial r}\left(\frac{1}{E^{(1)}} \cdots \frac{\partial}{\partial r}\left(\frac{1}{E^{(1)}} \frac{\partial}{\partial r}\left(\frac{r^{n-2}}{E^{(1)}} H f m\right)\right) \cdots\right) e^{i|X| E} d r
$$

where there are $(n-2) / 2$ differentiations in the integrand. If the differentiations are carried out, and the result simplified by cancellation, the integrand can be expressed as a sum of the form

$$
\sum \xi_{i} \frac{r^{u_{i}}}{\left(E^{(1)}\right)^{v_{i}}} e^{i|X| E}
$$

where for each $i, u_{i}, v_{i} \geqq(n-2) / 2$, and the $\xi_{i}$ are products of various derivatives with respect to $r$ of $f, E^{(1)}, H$, and $m$.

Now it is a simple matter to verify that in a given $\xi_{i}$, the number of factors which are derivatives of $E^{(1)}$ is precisely $v_{i}-(n-2) / 2$ and, moreover, if we take the order of each derivative of $E^{(1)}$ which appears as a factor, multiplied by the number of times it appears as a factor, and add up the results, the sum will be less than or equal to $u_{i}-(n-2) / 2$.

We now return to (13), with the remark that if $n=2$, the integrand is simply $H f m e^{i|X| E}$, and this case is covered in what follows.

Define $\delta=(|X| T(P, \varphi))^{-1 / 2}$, where $T(P, \varphi)=\sum_{i=1}^{n-1} \varphi_{i}^{2} p_{i}^{2 k-2}$.

Now $\int_{0}^{\varepsilon}=\int_{0}^{\delta}+\int_{\delta}^{\varepsilon}$. Consider first the integral from 0 to $\delta$. (The case in which $\varepsilon<\delta$ is subsumed under our estimate for this integral.) Now if we examine a typical term in the integrand, it follows from Lemma 6, and our observations about the 
$\xi_{i}$ 's, that the integrand is bounded by a multiple of $\left(r / E^{(1)}\right)^{(n-2) / 2}$, and by Lemma 3, this is in turn bounded by $(T(P, \varphi))^{-(n-2) / 2}$. I.e.,

$$
\begin{aligned}
|X|^{-(n-2) / 2} \int_{0}^{\delta} & =O\left((|X| T(P, \varphi))^{-(n-2) / 2} \delta\right) \\
& =O\left((|X| T(P, \varphi))^{-(n-1) / 2}\right) .
\end{aligned}
$$

If, now, we integrate with respect to $\varphi$ and invoke Lemma 7 , and recall that $p_{i}=c\left(x_{i}^{*}\right)^{1 /(2 k-1)}$, we see that the result is $O\left((A(X))^{-(k-1) /(2 k-1)}|X|^{-(n-1) / 2}\right)$.

To deal with the integral from $\delta$ to $\varepsilon$, consider a typical term, i.e.,

$$
\frac{\text { constant }}{|X|^{(n-2) / 2}} \int_{\delta}^{\varepsilon} \frac{\xi_{i} r^{u_{i}}}{\left(E^{(1)}\right)^{v_{i}}} e^{i|X| E} d r
$$

Now

$$
\begin{aligned}
\frac{1}{|X|^{(n-2) / 2}} \int_{\delta}^{\delta} \frac{\xi_{l} r^{u_{i}}}{\left(E^{(1)}\right)^{v_{\imath}}} e^{i|X| E} d r & =\frac{1}{i|X|^{n / 2}} \int_{\delta}^{\varepsilon} \frac{\xi_{i} r^{u_{i}}}{\left(E^{(1)}\right)^{v_{\imath}+1}} d\left(e^{i|X| E}\right) \\
& =\left.\frac{-i|X|^{-n / 2} \xi_{i} r^{u_{i}} e^{i|X| E}}{\left(E^{(1)}\right)^{v_{i}+1}}\right|_{\delta} ^{\varepsilon}+i|X|^{-n / 2} \int_{\delta}^{\varepsilon} \frac{\partial}{\partial r}\left(\frac{\xi_{i} r^{u_{i}}}{\left(E^{(1)}\right)^{v_{i}+1}}\right) e^{i|X| E} .
\end{aligned}
$$

As before, it follows from Lemmas 3 and 6, and our observations about the $\xi_{i}$ 's, that the first term is $O\left((|X| T(P, \varphi))^{-(n-1) / 2}\right)$, and similar considerations show that the integrand in the second term is $O\left((T(P, \varphi))^{-n / 2} r^{-2}\right)$. From this it follows that the second term is $O\left((|X| T(P, \varphi))^{-(n-1) / 2}\right)$ and, as before, this implies that the result of integrating both terms with respect to $\varphi$ is $O\left((A(X))^{-(k-1) /(2 k-1)}|X|^{-(n-1) / 2}\right)$, which completes the proof of Theorem 2 .

\section{REFERENCES}

1. A. Erdélyi, Asymptotic expansions, Dover, New York, 1956.

2. Walter Littman, Fourier transforms of surface-carried measures and differentiability of surface averages, Bull. Amer. Math. Soc. 69 (1963), 766-770.

3. B. Randol, A lattice-point problem, Trans. Amer. Math. Soc. 121 (1966), 257-268.

4. J. G. van der Corput, Over roosterpunten in het platte vlak, Noordhoff, Groningen, 1919.

YALE UNIVERSITY,

New Haven, Connecticut 